THEORIA ET HISTORIA SCIENTIARUM, VOL. VI, N²

Ed. Nicolas Copernicus University 2002

Ronnie Brown, Timothy Porter

\title{
Mathematical Structure: Knowledge, Representation and Interpretation
}

\begin{abstract}
The main theme of this article is to show the abstraction processes of mathematics allow hierarchies of structure, in which for example a 'comparison' becomes an object and so allows for methods of comparison themselves to be compared. Further, a representation of an object need not be unique, and the comparison of representations is important for our understanding and use of an abstraction process.
\end{abstract}

\section{Introduction}

In 1989 the Pop Maths Roadshow started at Leeds University and then toured the UK. For this, the authors and Nick Gilbert designed and produced a travelling exhibition of sixteen A2 boards entitled 'Mathematics and Knots'. (This is now available in electronic form at the website: http://www.bangor.ac.uk/ma/CPM/ exhibit/) The main aim of our exhibition was not simply to explain knot theory to the general public, but instead to examine at a popular level various

methodological themes in mathematics using elementary parts of knot theory as an illustration. Thus a more accurate title might have been 'Mathematics through Knots'.

We have discussed our processes and 'philosophy' in two articles: one, Brown and Porter, 1990, on the actual processes involved in the building of our mathematical exhibition; the other, Brown and Porter, 1995, on our views on how an examination of mathematical methodology can, and probably should, influence the presentation of mathematics not only to our students and other

young people, but also in our attempts to convey mathematics to the general public and to fellow scientists. 
We will examine some of these methodological themes in this article, since they are not exclusive to mathematics and fit with the overall theme of this volume.

What were these themes? They included Representation, Classification, Invariants, Abstraction, Analogy, the breaking down of objects and processes into 'elementary 'parts, and the rules by which these parts are combined. For this article we would stress one further theme that is behind several of these, namely Comparison / Interrelation. Without a means of Comparison, without interaction between the objects of study, there can be little chance of Classification. Comparison is the essence of the use of Invariants. If two objects have different features, are they 'of the same type' or 'different'? It of course depends on your comparison, but how does one compare? Finally for an arbitrary object to be broken down as a composite of simpler 'elements' implies that the 'elements' can be compared with the object in the first place. Beware, however, comparison is not always possible. Within the popular media presentation of Science, no recognition seems to exist that two objects may be related to a third without themselves being related. This mistake is also current in many branches of science itself!

\section{Themes}

We have already discussed the above themes in the article Brown and Porter, 1990, and illustrated them in the exhibition and website through the medium of knots. Here we will use a number of different illustrations based around natural, that is, whole, numbers. The familiarity of this example will aid us in pointing out features relevant to our themes.

\section{(a) Classification}

There are several 'classifications' of numbers used in everyday life. The simplest is 'even' (i.e. divisible by 2) and 'odd' (not so divisible, and so leaving remainder 1 on division by 2), but we could also mention prime or non-prime, and the classification according to size which can be more vague (large, small, etc.)

In each classification, some comparison is used. For 'prime/non-prime', it is the invariant 'primeness'; in that of size it is the relationship, $a \leq b$, which is read $a$ is less than or equal to $b$; and in the even/odd case it is divisibility by 2 .

\section{(b) Comparison}

In (a) we used ' $a \leq b$ '. This is an 'order relationship' less than or equal. A classification could also be done using other similar relationships. 
Suppose $a, b$ are numbers. We could ask 'does $a$ divide $b$ ?' by which we mean is there some $c$ such that $a c=b$. It will be helpful to abbreviate ' $a$ divides $b$ ' to 'a $\mid \mathrm{b}$ ', so for instance $2 \mid 6$ but $2 / 5$ (2 does not divide 5). Again $a \mid b$ has some properties that are very similar to those of $a \leq b$. So we note:

(i) $a \mid a$ for any $a$ (compare with $a \leq a$ for any $a$ )

(ii) $a \mid b$ and $b \mid a$ implies $a=b$ (Remember $a, b$ are natural numbers and so are positive.)

(iii) $a \mid b$ and $b \mid c$ implies $a \mid c$

Mathematicians give a name to relations satisfying these properties: they are called order relations. One difference between this relation and ' $a \leq b$ ' is that for any $a, b$, it must be true that either $a \leq b$ or $b \leq a^{\prime}$ but we noted that $2 \longdiv { 5 }$ and of course $5 / 2$.

[Reflection; In 'popular' language, especially that employed in the 'media', all order relations are 'linear' (i.e. like $a \leq b$ for numbers $a, b$ ), so if there are two tennis players or football teams one must be better than the other. This even extends to 'popular' discussions of scientific theories. One even hears radio or television presenters saying that it is 'logical' that one must be better than the other. Sometimes scientists themselves are 'guilty' of this, in claiming for example that one animal is better adapted for some particular ecosystem than another when they may not be 'comparable'.]

In our discussion of these comparisons, it is clear that a process of abstraction is occurring. We are in fact considering the means of comparison (technically the relation, $\mid$, or $\leq$ ) as a thing in itself. We are comparing the comparisons. This is an important point.

Paton, 1997, in his discussion of glue, verb and text metaphors in Biology, has pointed out that science frequently uses 'glue' and particular verbs to emphasise the interrelations between objects. He gives the following quotation:

distributed decentralised network is more a process than a thing. In the logic of the Net, there is a shift from nouns to verbs. Economists now reckon that commercial products are best treated as though they were services ...It is not what something is, it s what it is connected to, what it does. Flows become more

important than resources... Kelly (1994) p. 27

Here our elementary discussion illustrates a mathematical exemplar, but the means of comparison 'things' is not a 'thing' nor a 'process' it is both. Like the particle/wave duality of quantum physics, our order relations help in the classification of objects (so are 'verblike') but are objects in their own right (i.e. 'thing' like). To use the economic example above a flow of goods is an object. Turning a relation into an object is the method at the heart of abstraction. 


\section{(c) Abstraction and Representation}

We have already 'abstracted' our division-order relation from being a 'verb' to a 'noun'. The question of its representation is easily handled using an idea of H.Hasse. We draw a network with nodes corresponding to the numbers and an upwards line from $a$ to $b$ if $a \backslash b$. The resulting network will be infinite as there are infinitely many natural numbers, so we will restrict attention to the subnetworks corresponding to the sets of divisors of particular numbers. For instance the set of divisors of 18 is $\{1,2,3,6,9,18\}$ and we get that the order relation has Hasse diagram.

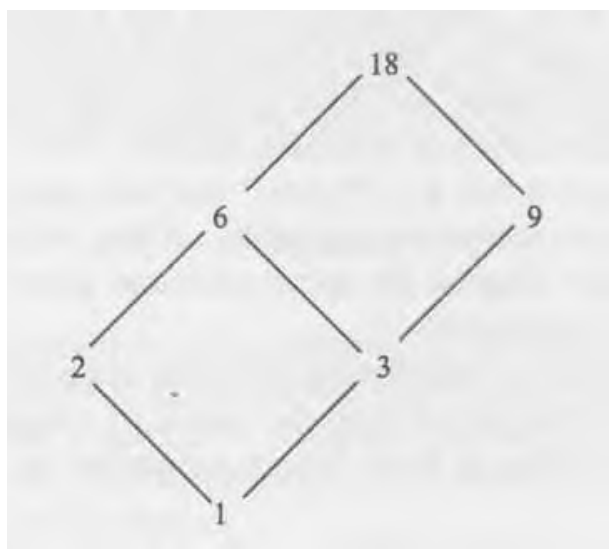

Why is this a good representation of the information? For a start it is fairly simple visually, but contains essential information about 18 and its divisors. It shows that 2 and 3 are not comparable; it shows that there is internal 'structure' in the divisor relation such as

(i) given any two nodes, there is a common node above them and, in fact, a 'least' such node;

(ii) given any two nodes, there is a common node below them and, in fact, there is a 'greatest' such node.

These correspond to 'least common multiple' $(1 \mathrm{~cm})$ and 'greatest common divisor' (gcd). Their existence is a special property of certain order relations.

(iii) there is a bottom and top element;

(iv) there are some 'atoms', that is, elements which are immediately above the bottom element. (Of course, these are the prime factors of the number and we will return to them later.)

A common criticism of mathematics is that in its examples it does not look at complicated enough situations or on the other hand that it makes simple things 
too complicated itself. Here one needs to examine the sense of 'complication'. We have used a simple example and everything is easily seen and understood in this small diagram. If we had taken a more complicated example could we have understood it more simply?

Take for instance 2075, a number of moderate size. The divisors of 2075 are $\{1,5,25$, $83,415,2075\}$ and, of course, the diagram looks very much the same. There are thus properties of 18 and 2075 that are similar. We have found them by examining the Hasse diagrams for their sets of divisors. Other numbers may give different forms of diagram. For instance 64 also has 6 divisors, but a different Hasse diagram. The number 30 has $\{1$, $2,3,5,6,10,15,30\}$ as its set of divisors and a cube as its Hasse diagram, but 31 has just

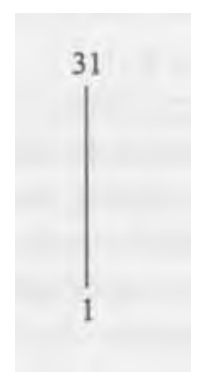

so 'complication' is not dependent on size alone. In each case, the diagram reveals some structure of the number, namely the parts of which it is made. Of course, if someone asks for the Hasse diagram of 180774 then the number of directions needed and the number of nodes needed starts to get in the way of the easy reading of the divisor information, since

$$
180774=2 \times 3^{2} \times 11^{2} \times 83
$$

The diagram is thus only the first step in the abstraction process. The features observed have then to be analysed more closely and abstractly.

\section{(d) Analogy}

Another feature of the abstraction process is that, having used the order relation "divides" to compare numbers, we can find analogues of this structure' elsewhere. An obvious example is to take a set, $X$, and look at all its subsets. This in turn gives us a new set (often called the power set of $X$ )

denoted $\rho(X)$. There is an obvious order relation on $\rho(X)$ namely "is a subset of': $A \subseteq B$ reads ' $A$ is a subset of $B$ ' so taking as an example $\mathrm{X}=\{1,2,3\}$ gives subsets of $X$ : 


\section{$\varnothing,\{1\},\{2\},\{3\},\{1,2\},\{2,3\},\{1,3\}, X$}

(Here, $\varnothing$ is the empty set \{\} having no elements. It is worth noting that mathematics may be accused of pedantry: it seems extraordinary to take the trouble to give a notation for an empty box! In fact this pedantry is important: mathematics was held up for centuries for lack of the 'trivial concept' of zero, which is a necessary foundation for the Arabic system of numeration.)

There are numerous features which 'subset of' has in common with the case of 'divides'. The basic structure is that of a set of things with a particular type of relation on it. Comparison suggests the following three are crucial to the common features: for any $\mathrm{A} \in \wp(X)$, that is for any subsets $A, B, C$ of $X$,

1. $A \subseteq A$,

2. $A \subseteq B$ and $B \subseteq A$ implies $A=B$.

3. If $A \subseteq B$ and $B \subseteq C$ then $A \subseteq C$.

Thus the set of subsets of $X$ with the relation of inclusion is what is called a partially ordered set. The partial order $\subseteq$ does not necessarily allow comparison of just any old pairs of subsets. That is why it is called 'partial'.

We can represent small examples of sets of subsets by Hasse diagrams in a way that helps present the information clearly. (The above example gives a cube just like the divisors of 30.) There are analogues for the least common multiple and greatest common divisor (given by union and intersection respectively).

In a partially ordered set, other concepts can be defined, though they may not always be applicable. Thus the complement of an element can be defined as: if $x$ is an element of some partially ordered set, a complement for $x$ is the smallest $y$ such that the 'least common multiple' of $x$ and $y$ is the top element. In the partially ordered set of divisors of 18 , the complement of element 9 in 2 since $1 \mathrm{~cm}\{2,9\}=18$, the top element. In the set of subsets of $\{1,2,3\}$, the complement of $\{1,3\}$ is, of course, $\{2\}$.

However, this does not make sense unless these least common multiples (usually called Terns') exist nor unless the partially ordered set has a top element. In other words, attempting to generalise a feature in observed cases, to a generic abstract case, focuses attention on the properties, i.e. the invariant features or conditions, that imply that the property holds. Here one is able to see the strength of abstraction at work. We note:

By abstracting, we get general ideas that can be applied outside the original setting especially using analogy.

In our two types of example, set complement is an obvious operation in $\wp(X)$, so one may look for its analogues elsewhere where a partially ordered set structure exists. Comparing the instances with the abstract gives a sort of 'technology transfer'. 
Our examples were simple: 'divisors' and 'subsets' and comparisons within these settings, in the form of relations ( $a \mid b$ or $A \subseteq B$ ), gave structure not initially apparent. Comparing the structures yields common features but also some differences. (Any partially ordered set of the form $(\wp(X), \subseteq)$ for $X$ finite can also be found amongst the divisor partially order sets $\left(\operatorname{Div}_{n}, \mid\right)$ but the partially ordered set $\left(\operatorname{Div}_{18}, \mid\right)$ has 6 elements, whilst all $(\wp(X), \subseteq)$ have a power of 2 as their size.)

\section{Abstraction makes comparison easier as special features of examples are blurred}

The discussion above compared $(\wp(X), \subseteq)$ with $\left(\operatorname{Div}_{n}, \mid\right)$ without reference to the fact that the elements of $\wp(X)$, are subsets of $X$ and those of $\operatorname{Div}_{n}$ are numbers. In mathematics analogies are fundamental, but we make analogies not between things but between the relations between things. It is the abstract structures which have analogous properties.

\section{Remarks}

The structure of these two types of examples is that of what is known as a 'lattice'. Other examples can be found such as those coming from logic. The properties of the existence of lcm's $x \vee y$ and greatest common divisors (called 'gcd's') $x \wedge y$ give extra structure. The possibility of relaxing properties of these leads to variants that capture different features and 'damp down' others. The variant structures then can be used to model new (and highly significant) situations. The importance of abstraction here is that the features of examples are not logically independent. The obvious example being that in the presence of a complement operation, $x \vee y$ and $x \wedge y$ are derivable one from the other, giving an abstract version of de Morgan's laws. The use of analogy extends further. The notation is chosen to reflect the analogy.

The notation $x \vee y$ suggests union $A \cup B$, whilst $x \wedge y$ suggests intersection $A \cap B$, and of course $\leq$ and $\subseteq$ are related though we will not go into the historical question of which came first and when. Notice that mathematicians like to use notations which are themselves metaphors.

It is noticeable that the fact that the concepts can be represented by diagrams has been formative. We will see shortly that diagrammatic representation does have limitations and progress and interpretation beyond those limits is consequently slower. 


\section{(e) Indecomposables}

The operations of gcd (and $1 \mathrm{~cm}$ ) give a way of 'composing' numbers as do, more simply, multiplication and addition. If we can 'compose', can we 'decompose'? Of course we can and the ways of decomposing are exactly what the divisor lattices show. We can build up a number from simpler/smaller bits. Some numbers are 'indecomposable'. You cannot split them further. These are of course the prime numbers and they have the simple divisor structure given by the left hand picture below:
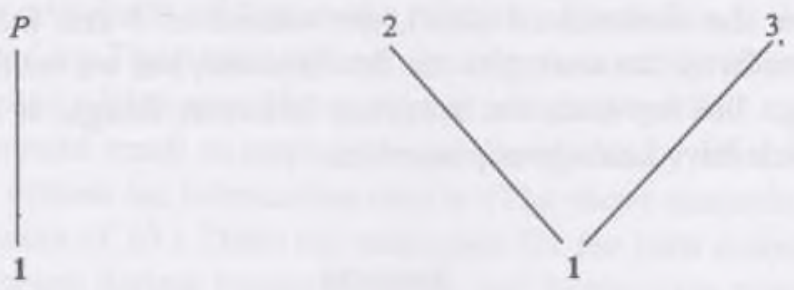

In our examples of divisor lattices, the primes are the parts growing out of 1 , eg. in the set of divisors of 18 we have as in the right hand picture above.

Of course, the number of distinct primes determines the 'dimension' of the diagram used (18 has 2 prime factors so the diagram is two-dimensional, 64 has only one prime

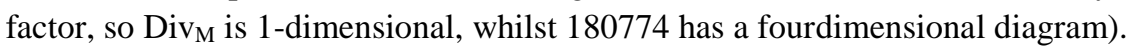

\section{Analogues of the primes in our power set examples are the single element} sets, so that for $X=\{1,2,3\}$ the indecomposables in $\wp(X)$ are $\{1\},\{2\}$, and $\{3\}$.

Of course the notion of indecomposables or atoms can be abstracted and analogues can be found in other settings, but no decomposition of general elements need exist and analogues with the primes may fall apart. The reasons why are very relevant to our discussion but we will need some further development before we can return to this.

We argued for the duality 'verb/thing', so can we decompose 'verbs' i.e. comparisons, into simpler parts. What can this mean? In our examples it is relatively easy to see.

We have in Div lgthat $2 \mid 18$ is "related to" 18 . The relation is not as simple as some others. We have $3 \mid 9$ and the portion of the Hasse diagram we need is 


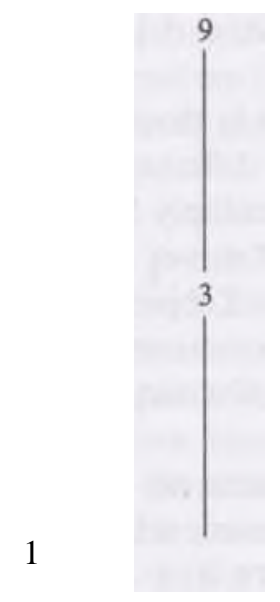

which is a copy of the diagram on the left below
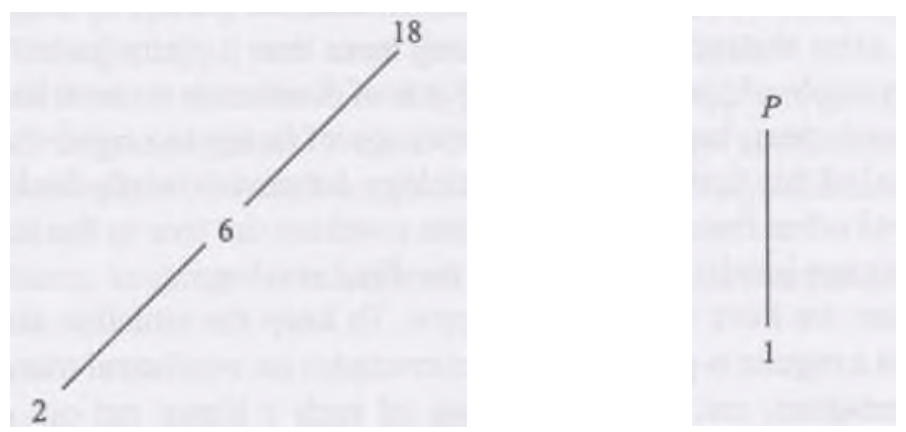

for the divisors of 9 . Of course, we can see why. If we divide 9 by 3 we get 3 which is prime, but if we divide 18 by 2 we get 9 (and a copy of Divg).

We could thus decompose the relationship $a \mid b$ into a series of smaller comparisons

$$
a \backslash b_{r} b^{\wedge} b^{\wedge} \cdot-. b^{\wedge} b
$$

where each comparison is a copy of the atomic Hasse diagram for a prime $p$ which we gave above, on the right. These comparisons give the prime decomposition of the quotient $c=b / a$.

We have some interesting features even in this simple case of the divisors of 18 . The decomposition of 3 | 18 is not unique. We could go 
$3 \mid 9$ and $9 \mid 18$, or $3 \mid 6$ and $6 \mid 18$.

So we have two different routes in the diagram from 3 to 18 . They seem in some ways equivalent, but they are different. We have two different "moves" - one is multiply by 2 , the other is multiply by 3 . It happens that the end result does not depend on which order the moves are made since $2 \times 3=3 \times 2$.

Summarising, the decomposition of objects into irreducible or indecomposable pieces need not stop there. We can sometimes decompose comparisons, reducing some comparison to a composite of a sequence of "elementary moves", i.e. indecomposable comparison.

The 'comparisons' between objects are often called 'morphisms', so as to suggest that they are compatible with some internal structure or shape of the objects they compare. In a partial order, there is no more than one comparison between objects, but there could be other situations where there are several comparisons, for example each could be a decision process for a comparison. A key point is that the set of 'morphisms' between the numbers in the set $\operatorname{Div}_{\mathrm{n}}$ of divisors of $n$ can itself have structure. This suggests a hierarchy of structures with 'morphisms' between 'morphisms' and so on. This is often the case, but why should we bother with such extra abstraction. Is it anything more than a pretty game?

Our example of (partially) ordered sets of divisors as an invariant of numbers is simple and clear, but has the disadvantage of being too rigid. Before leaving the 'themes' of this first level of methodology, let us very briefly look at a situation with several other features. We will then combine the two in the next section to examine higher levels of structure in the final section.

Suppose we have a geometric figure. To keep the situation simple we will assume it is a regular n-gon so particular examples are equilateral triangles, squares, regular pentagons, etc. A simple image of such a figure cut out of wood and fitting in a hole of the same shape and size may help, i.e. a children's toy. The figure can be lifted, turned around or turned over and replaced in its hole. If there are n-sides this yields $2 n$ positions in which we can put the block. These positions are the objects we want to study so we will naturally be looking for 'comparisons' between them. As an illustration look at an equilateral triangle on the left:
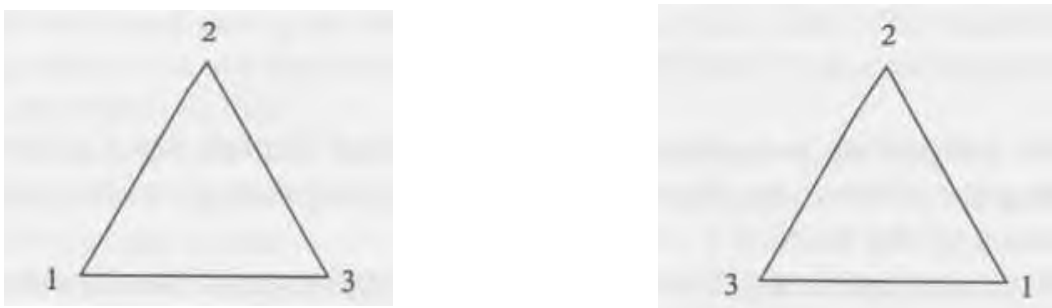
Labelling the vertices (and the 'vertices' of the whole) is a natural thing to do and with this labelling the comparison between the standard position and that obtained by flipping over the block giving the right hand figure might be represented by a symbol

yielding what is called a 'permutation' of the vertex labels 1, 2, 3 .

Applying our methodology, we have already represented our situation symbolically. There are various invariants of these positions that aid in a classification of the positions. For instance, two positions have the same orientation if reading off the labels 1 then 2 then 3 you go the same way around the hole. (The above two have different orientation.) This classifies the positions into two classes, which might be called 'clockwise' and 'anticlockwise' positions. There are two obvious elementary moves: at any position, pick the n-gon up, rotate it through $360^{\circ} /$ n (i.e. one 'notch' clockwise) and put it back (an elementary rotation) or pick it up, flip it about a fixed axis of symmetry (in our case of the triangle the axis through 2 was used above), and put it back.

It is well known that these two types of elementary moves generate all comparisons between positions. We can thus represent any 'comparison' between positions by a sequence of such moves. However such a representation will not be unique.

For instance, keeping the triangle as our example, the comparison

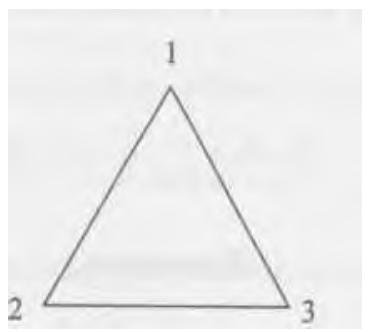

can be decomposed as rotate, rotate again, then flip or as flip then rotate. Writing $R$ for rotate and 5 for flip:

$$
R^{2} S=S R
$$

The idea of identifying simple component 'moves' allows one to represent complex processes as composites of simple ones. This helps in the understanding of those more complex processes by representing and interpreting those processes. 
The resulting representation is however not always unique, as we saw above. At first sight this might seem to be a disaster since if different representations of the same complex process exist, it may be difficult to decide which one we should we use. The point is more that for sufficiently precise formulations of concepts, we would expect a mathematical theory which would enable us to make such decisions. This is in fact a significant theory, called rewriting theory, and it leads to questions of which rewriting problems are decidable, what are the algorithms for making decisions when such can be made, and how efficient and usable they can be made.

The more optimistic and valid view is that such multiple representation of the complex process incorporates a wealth of higher order information, but this information needs more advanced techniques to handle it, in fact deeper algebra.

We thus find that abstraction, comparison, interpretation and representation interact at a fairly early stage in such situations. To understand this more deeply we need some new concepts and examples of them as our existing examples are slightly too rigid for the more general points we need to examine.

\section{Categories}

We have been repeatedly using comparisons between objects as a key concept in our methodology. The concept of a category is an abstraction of this context.

We suppose we have a collection of objects and comparison morphisms between them. We will use letters $A, B, \ldots, X, Y$ etc for the objects and labelled arrows for comparisons

$$
X \stackrel{f}{\rightarrow} Y \quad a \stackrel{\varphi}{\rightarrow} b
$$

\section{Examples}

- The objects might be the divisors of 18 and the arrows represent the fact that $a \mathrm{~V}$ (but written in the form $a \longrightarrow b$.

- The objects might be positions of a regular «-gon and the arrows the ways of going from one to another.

- The objects might be sets and the arrows would represent functions between the sets.

- The objects might be sets and the arrows represent relations between them. (A relation $R$ from a set A to a set $Y$ is a set of pairs (x,y) with $x$ in $X, y$ in $Y$. If $(\mathrm{x}, \mathrm{y})$ is in $R$ we think of this as being ' $\mathrm{x}$ is related to $\left.y^{\prime \prime}\right)$. 
- The objects might be partially ordered sets with arrows representing functions between them which are compatible with the order. ( $\mathrm{f}: X \rightarrow Y$ is compatible with the order if $x_{1} \leq x_{2}$ implies $f\left(x_{1}\right) \leq f\left(x_{2}\right)$.).

(In general if we have a collection of sets having the same type of structure, we can choose arrows to represent functions that preserve or are compatible with that structure in some way.)

In each of these examples, the objects and arrows interrelate. Any arrow starts at some object and finishes at an object (which may be the same or different). If we have an arrow from $a$ to $b$ and another from $b$ to $c$ we can compose them and get one from $a$ to $c$

$$
a \stackrel{f}{\rightarrow} b \stackrel{g}{\longrightarrow} c \text { yields } a \stackrel{\text { \& }}{\longrightarrow} c
$$

We also always have for each object a special identity arrow starting and finishing at that object. For instance if $a$ is a divisor of 18 then of course $a \mid a$ is true, and we have an identity arrow from $a$ to itself.

The structure sketched out above is that of a 'category'. It is a setting designed almost explicitly for comparing objects. We will not explore it in detail here but will examine the way in which analogues of some of the features that were observed earlier in Section 2 can be formulated.

Analogues of $\mathrm{x} \wedge \mathrm{y}$

In the set $\operatorname{Div}_{n}$ of divisors of the number $n, x \wedge y$ is interpreted as being the greatest common divisor of the two elements $x$ and $y$.

In the set $\wp(X)$ of subsets of $X$ the set $x \wedge y$ is just the intersection of the two subsets labelled $x$ and $y$.

In both cases if we have any other 'object' $z$ and comparison arrows

$$
z \rightarrow x \text { and } z \rightarrow y
$$

then there is a uniquely determined comparison arrow

$$
z \rightarrow x \wedge y
$$

We can do this more generally. Suppose we have a 'diagram', that is a collection of objects and perhaps some arrows between them. Then we can sometimes find an object which is the "greatest" one with arrows to all objects of the diagram. (The resulting object is an example of what is called a limit of the diagram. See any book on category theory for the exact definition.) 


\section{Analogues of $x \mathrm{v} y$}

In the set of divisors of $t$ the element $x \mathrm{v} y$ was the least common multiple of the numbers $x$ and $y$.

In the set $\rho(X)$ of subsets of $X$, the element $x \vee y$ is the union $x \cup y$ of the subsets $x$ and $y$.

The analogue and generalisation for a diagram of interacting objects in a category is called a colimit.

\section{Remark}

Colimits have been used to good effect to describe biological hierarchical systems (cf. Ehresmann and van Vanbremeersch, 1987). They allow one to model mathematically the idea that the whole is greater than the sum of its parts.

Following our themes we should expect to consider 'comparisons' between categories (these are called 'functors'). Moreover there are comparisons between functors, so that given categories $C$ and $D$, there is a category whose objects are the functors from $C$ to $D$. This yields a hierarchy of structures known as $n$-categories which have recently been finding application in parts of theoretical physics. How do these higher order versions of categories reflect the other themes?

We will restrict our attention to two themes, those of breaking down into 'elementary' parts and representation. We will illustrate with ideas from the theory of categories. When we looked at $\operatorname{Div}_{\mid g}$ the elementary parts were fairly obvious, they were the primes, 2 and 3. It is often not that obvious what 'irreducibles' or 'indecomposables' to use for building blocks. For our discussion we will assume a collection of arrows in the category $C$ has been selected and we have checked that it has the property that any arrow in $C$ can be written as a composite of these special arrows, although of course we would not expect all arrows to be special. We saw this in our example of moves on a triangle (or more generally an «-gon). There every arrow could be written as a composite of those arrows that from any position corresponded to an elementary rotation or a flip about the vertical axis of symmetry, but not all,arrows from the position would have that form.

A choice of special arrows being made we can represent any arrow by a symbol consisting of a list of the special arrows yielding the arrow on composition. This representation is an algebraic 'icon' allowing manipulation, calculation etc. concerning the arrow so is a powerful help in the study of the situation, however it is usually not unique.

We summarise in general: to

obtain a representation, 
(i) choose some 'elementary' special objects that composed or combined suitably will generate all the objects of interest,

(ii) for each specific object, construct a list whose elements are special objects and whose composite or combination is the given specific object. (This is subdivision of objects.) There are then two generic problems

a) what would be the consequence to the representation of choosing a different set of special objects

and, even with just one such set,

b) there may be many lists that have the same composite.

We saw problem b) with our example of a triangle as

$$
R^{2} S=S R
$$

so the lists $(\mathrm{R}, R, S)$ and $(S, R)$ yield the same arrow on composition.

There is another more subtle point: lists may not suffice. For instance if we have a twodimensional tiling pattern, it is clearly made up of the tiles as elementary parts but the way the parts fit together are too subtle to be simply given by a list - we need some sort of 2dimensional listing or gluing information. The study of the ways that one replaces 'lists' by higher order structures then reveals a lot about the (representation of the) original object.

Returning to our categorical examples, we find that between two objects there may be many different lists yielding the same arrow, so one tries to see if the set of such lists has some structure itself so that perhaps there are elementary 'rewriting rules' that allow one list to be changed to another. Ideally one hopes

that if two lists yield the same arrow then it will be possible to get from one to the other by simple application of the rewriting rules. These rewriting rules constitute another level of the hierarchy of structure.

The interpretation of the rewriting rules in terms of the original structure is often very subtle.

Again a summary seems necessary.

(iii) A representation of objects may not be unique. If it is not, then its lack of uniqueness needs study.

(iv) The reasons why two representations yield the same object yield information on the object. These reasons are often themselves structured.

\section{Epilogue}

We have tried to suggest how representation of objects or processes in terms of more elementary building blocks naturally leads to a hierarchy of structures. 
The use of such hierarchies is well established in parts of mathematics and by the use of metaphor in the guise of mathematical modelling this hierarchy of structures can lead to a deeper understanding of the linkage between the model (i.e. the representation) and the processes being modelled (i.e. the interpretation).

Throughout we have made use of analogy and abstraction as essential tools in the development of the ideas and most crucially we have stressed the need for well structured notions of comparison.

\section{References}

Brown, R. and Porter, T., 1989, Why we made an exhibition, in Souvenir Guide to the Pop Maths Road Show, Royal Society/ Joint Mathematics Council 10-12.

Brown, R. and Porter, T., 1990, Making a mathematical exhibition, in The popularization of mathematics, edited A. G. Howson and J.-P. Kahane, ICMI Study Series, Cambridge University Press, 51-64.

Brown, R. and Porter, T., The methodology of mathematics, Math. Gazette, July (1995) (1995) 322-335.

Ehresmann, A. And Vanbremeersch, J.-P., 1987, Hierarchical Evolutive Systems: a mathematical model for complex systems, Bull of Math. Biology, 549, 13-50.

Kelly, K., 1994, Out of control: The new biology of machines, London, Fourth Estate. Paton, R., 1997, Verbs and.Text metaphors in biology, Acta Biotheoretica, 45, 1-15. 